\section{Komplette endovaskuläre Rekonstruktion der Viszeralarterien}

Die am häufigsten eingesetzte Therapie von Verschlüssen der großen Abdominalgefäße im Rahmen der chronischen mesenterialen Ischämie stellt $\mathrm{z}$. Zt. die gefäßchirurgische Operation dar (Sandmann W et al., Dtsch Med Wschr 1994, 119 :979). Die Platzierung von Stents gewinnt zunehmend an Bedeutung (Sheeran SR et al., J Vasc Intervent Radiol 1999; 10: 861), insbesondere bei Patienten, deren Operationsfähigkeit eingeschränkt ist. Die alleinige PTA der genannten Gefäße hat sich zu einem gewissen Grade etabliert. Trotz der Vorteile dieser wenig invasiven Methode im Vergleich zur Operation sind es insbesondere die im Vergleich zur Gefäßchirurgie schlechteren Langzeitergebnisse, die die PTA nicht zur Therapie der ersten Wahl werden ließ. Eine neuere Alternative zur Versorgung solcher Verschlüsse stellt die Platzierung von Stents dar. Aufgrund der noch geringen Erfahrungen existieren $z$. Zt. noch keine einheitlichen Empfehlungen für die primäre Versorgung von Abdominalgefäßverschlüssen mit Stents.

Im Folgenden berichten wir den Fall eines symptomatischen Patienten mit einem Verschluss von Truncus coeliacus und A. mesenterica superior, der durch die Implantation zweier Palmaz-Stents erfolgreich behandelt werden konnte.

\section{Fallbeschreibung}

Ein 52-jähriger Patient stellte sich wegen postprandialer, epigastrischer Schmerzen mit Gewichstverlust und Dyspnoe vor. Keine Besserung auf orale Nitratgabe. Anamnestisch bekannt waren: Z. n. Posterolateralinfarkt, arterielle Hypertonie, Diabetes mellitus Typ II, Nikotinabusus, Adipositas, Hypercholesterinämie sowie eine Nierenagenesie rechts. Im Aufnahmestatus vorgealterter Patient, kein Herzgeräusch, Puls regelmäßig 80/min, RR 170/80. Geblähtes Abdomen mit Druckschmerz im linken Oberbauch und normalen Darmgeräuschen. Keine Ödeme, periphere Pulse regelrecht. Kein Fieber. Im Aufnahmelabor: 11500 Leukozyten pro $\mu$, CRP $5,2 \mathrm{mg} / \mathrm{dl}$, Amylase $62 \mathrm{U} / \mathrm{l}$, Fibrinogen $501 \mathrm{mg} / \mathrm{dl}$. - CK, LDH und Lactat im Normbereich. Unauffälliges EKG, Sinus- rhythmus. Keine wegweisenden Befunde im Röntgen-Thorax und der AbdomenSonographie. Verbesserung des klinischen Zustandes durch Infusion von Buscopan.

Zwei Tage nach der Aufnahme erfolgte eine Abdomen-CT ( $8 \mathrm{~mm}$ Schichtdicke, $12 \mathrm{~mm}$ Tischvorschub und $7 \mathrm{~mm}$ Inkrement, $120 \mathrm{ml}$ Ultravist 300 i.v.) im Sinne einer Screeninguntersuchung bei klinisch unklarem, abdominellen Krankheitsbild. Es zeigte sich eine $12 \mathrm{~cm}$ lange Wandverdickung des Ileums. Relevante Gefäßveränderungen fielen in dieser Untersuchung nicht auf.

Die Buscopantherapie unter der Arbeitsdiagnose einer Nierenkolik erzielte immer nur kurzfristigen Erfolg. Es kam immer wieder zu abdomineller Schmerzsymptomatik und weiterem Gewichtsverlust um $5 \mathrm{~kg}$. An weiterführender Diagnostik wurden eine Gastroskopie, eine Coloskopie und ein Dünndarmeinlauf nach Sellinck durchgeführt. Dabei zeigt sich eine Refluxösophagitis $\mathrm{I}^{\circ}$, umschriebene Schleimhautrötungen und ein fibrinbedecktes Ulcus im Colon ascendens; der Dünndarm stellte sich unauffällig dar. Kein Anhalt für Malignität. Die weitere Therapie unter der Hypothese einer entzündlichen Darmerkrankung oder einer Ulcuskrankheit führte zu keiner anhaltenden Besserung. Nunmehr unter dem Verdacht einer Mesenterialischämie wurde eine Gefäßdarstellung gewünscht. Wegen eines zwischenzeitlich eingetretenen Kreatinin-Anstieges auf $1,5 \mathrm{mg} / \mathrm{dl}$ etwa drei Wochen nach Aufnahme des Patienten wurde eine MR-Angiographie der Mesenterialarterien mit Gadolinium-DTPA durchgeführt. Es fanden sich ein $2 \mathrm{~cm}$ langer Abgangsverschluss der A. mesenterica superior mit Darstellung einer kräftigen Riolan-Anastomose und ein kurzstreckiger Verschluss des Truncus coeliacus sowie Kalkplaques am Abgang der A. mesenterica inferior. Angesichts der internistischen Vorerkrankungen des Patienten wurde in Absprache mit den Gefäßchirurgen die Indikation zur Angiographie mit Stentimplantation gestellt (Abb.1). Ein 8-French Renal Double Curve I Führungskatheter wurde über die linke A. femoralis eingebracht.

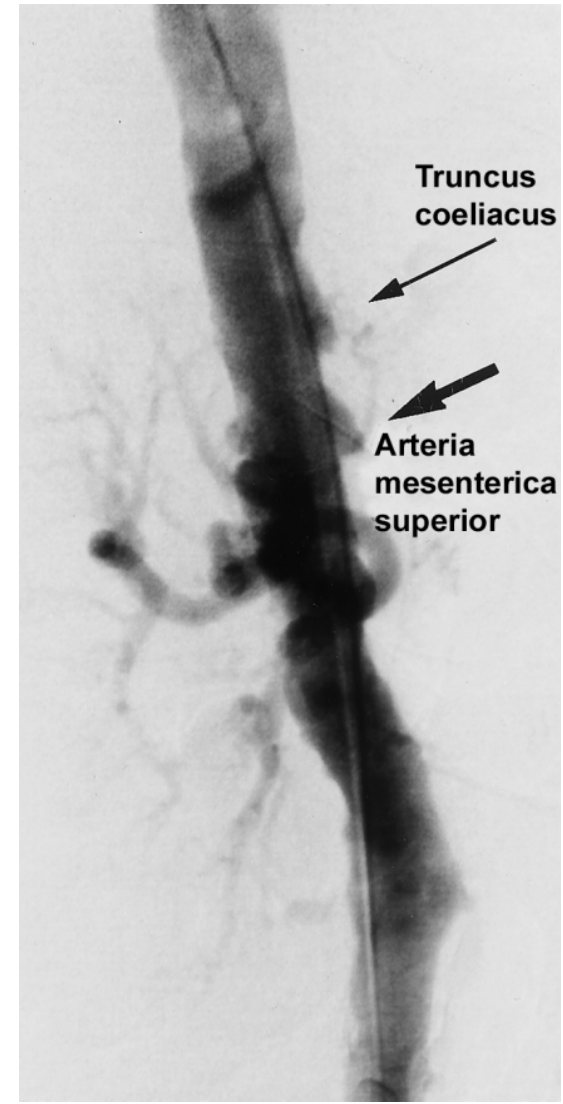

Abb. 1 Konventionelle Angiographie der großen Abdominalgefäße vor Stentimplantation. Verschluss von Truncus coeliacus $(\rightarrow)$ und A. mesenterica superior $(\rightarrow)$.

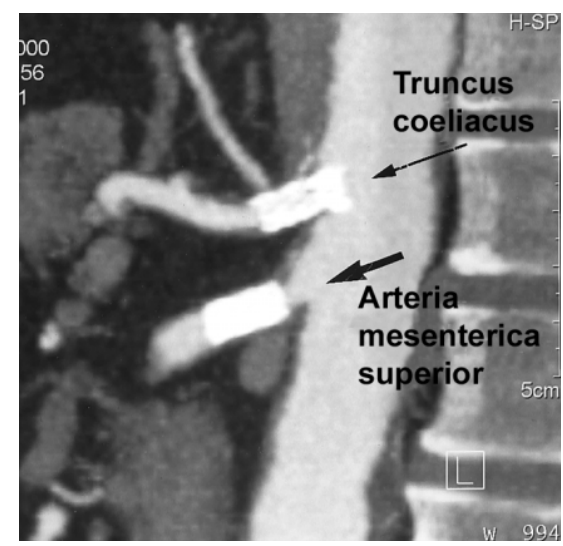

Abb. 2 Angio-CT: Zustand 41/2 Monate nach Intervention: Korrekte Lage der Palmaz-Stents in Truncus coeliacus $(\rightarrow)$ und A. mesenterica superior $(\rightarrow)$ und vollständige Offenheit beider Gefäße.

Unter üblichen Begleitmaßnahmen (5000 I.E. Heparin i.a., 50 mg Dolantin und $2 \mathrm{mg}$ Dormicum i.v.) wurden je ein $6 \mathrm{~mm}$ weiter und $18 \mathrm{~mm}$ langer Edelstahl-Palmaz-Corinthian-Stent ${ }^{\mathrm{TM}}$ (PC-186-PPS) (Fa. Cordis, Haan) im pro- 
ximalen Stammbereich des Truncus coeliacus und der A. mesenterica superior platziert. Vor der Stentfreisetzung wurden die Gefäßverschlüsse auf $3 \mathrm{~mm}$ vordilatiert. Dies gelang ohne abdominelle Symptomatik, keine Reststenosen, keine Embolie.

Seit der Intervention besserten sich die abdominellen Beschwerden erheblich und der Patient nahm adäquat an Gewicht zu. Die vier Monate nach der Intervention durchgeführte Kontroll-Angio-CT zeigte die korrekt liegenden Stents mit vollständiger Offenheit beider Gefäße (Abb. 2). Der Patient ist nach wie vor in deutlich gebessertem Zustand.

\section{Diskussion}

Der vorliegende Fall zeigt, dass die Platzierung von Stents eine sichere und effektive Methode zur Behandlung chronischer Verschlüsse der großen Abdominalarterien darstellt. Insbesondere bei inoperablen Patienten ist hierin eine wertvolle zusätzliche Behandlungsoption zu sehen. Inwiefern diese Methode an die Stelle der konventionellen gefäßchirurgischen Operationen treten kann, wird von den noch $\mathrm{zu}$ ermittelnden Langzeitergebnissen abhängen. In der Arbeit von Sheeran et al. (Sheeran SR et al, J Vasc Intervent Radiol 1999, 10: 861) wurden zwölf Patienten mit chronischer mesenterialer Ischämie mit Stents behandelt. 8 dieser Patienten hatten eine hochgradige Stenose an einem der drei großen Gefäße, einer an zwei Gefäßen und drei Patienten einen Verschluss je eines der großen Gefäße. Ein Patient verstarb innerhalb des ersten Monats nach dem - zunächst gelungenen Eingriff an einem Darminfarkt. Die primäre Patency nach 18 Monaten betrug $74 \%$ und die sekundäre Patency nach 3 Jahren $83 \%$. Im Rahmen von Case Reports berichten auch Busquet und Waybill über die erfolgreiche Rekanalisierung von Abdominalgefäßen. Im ersteren Fall handelte es sich um eine hochgradige Abgangsstenose der A. mesenterica superior in Verbindung mit einem Verschluss der A. mesenterica inferior (Busquet J, J Endovasc Surg 1997; 4: 380) und im zweiten um eine hochgradige Stenose von Truncus coeliacus und A. mesenterica superior in Verbindung mit einem Verschluss der A. mesenterica inferior (Waybill PN und Enea NA, J Vasc Intervent Radiol 1997; 8: 1069).
Im vorliegenden Fall hat der Patient mit seinem sehr ausgeprägten Befund eines Verschlusses von Truncus coeliacus und A. mesenterlca superior sowie einer 70\%igen Stenose der A. mesenterica inferior bei schweren internistischen Begleiterkrankungen von dieser Methode erheblich profitiert. In Bezug auf Arteriendissektionen, Re- und Reststenosen zeigt sich das Stenting der PTA überlegen. Wir glauben, dass zukünftige Entwicklungen und weitere Erfahrungen mit dieser Technologie zu einer Ausweitung der Indikationen für primäres Stenting von Mesenterialarterien führen werden.

\section{Springende Punkte}

- Die Implantation von Stents ist eine sichere und effektive Methode zur Therapie von Viszeralarterienokklusionen.

- Die Stentimplantation ist bei nichtoder bedingt operationsfähigen $\mathrm{Pa}$ tienten der chirurgischen Therapie vorzuziehen.

R. Feuls, J. König, S. H. Duda, Tübingen 